\title{
Oral Capecitabine in Gemcitabine- Pretreated Patients with Advanced Pancreatic Cancer
}

\author{
Stefan Boeck ${ }^{a}$ Ralf Wilkowski ${ }^{c} \quad$ Christiane J. Bruns $^{b}$ Rolf D. Issels ${ }^{a, d}$ \\ Christoph Schulz $^{\mathrm{a}}$ Nicolas Moosmann ${ }^{\mathrm{a}}$ Dorit Laessig ${ }^{\mathrm{a}}$ Michael Haas ${ }^{\mathrm{a}}$ \\ Alexander Golfe ${ }^{\text {Volker Heinemann }}{ }^{a}$

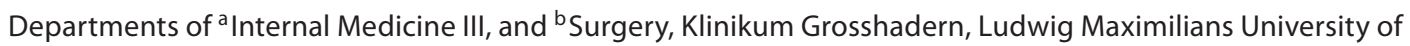 \\ Munich, Munich, 'Practice for Radiooncology and Radiotherapy, Klinik Bad Trissl, Oberaudorf, \\ ${ }^{d}$ GSF, National Research Center for Environment and Health, Neuherberg, and e Department of Medicine I, \\ Buergerhospital, Klinikum Stuttgart, Stuttgart, Germany
}

\section{Key Words}

Capecitabine $\cdot$ Chemotherapy $\cdot$ Gemcitabine $\cdot$

Pancreatic cancer

\begin{abstract}
Objective: To date, no standard regimen for salvage chemotherapy after gemcitabine (Gem) failure has been defined for patients with advanced pancreatic cancer (PC). Oral capecitabine (Cap) has shown promising activity in first-line chemotherapy trials in PC patients. Methods: Within a prospective single-center study, Cap was offered to patients who had already received at least 1 previous treatment regimen containing full-dose Gem (as a single agent, as part of a combination chemotherapy regimen or sequentially within a chemoradiotherapy protocol). Cap was administered orally at a dose of $1,250 \mathrm{mg} / \mathrm{m}^{2}$ twice daily for 14 days followed by 7 days of rest. Study endpoints were objective tumor response rate by imaging criteria (according to RECIST), carbohydrate antigen 19-9 (CA19-9) tumor marker response, time to progression, overall survival and toxicity. Results: A median of 3 treatment cycles (range 1-36) was given to 39 patients. After a median follow-up of 6.6 months, 27 patients were evaluable for response: no complete or partial respons-
\end{abstract}

\section{KARGER}

Fax +4161306 1234 E-Mail karger@karger.ch www.karger.com (c) 2008 S. Karger AG, Basel

0030-2414/07/0734-0221\$23.50/0

Accessible online at:

www.karger.com/ocl es were observed, but 15 patients (39\%) had stable disease. A CA19-9 reduction of $>20 \%$ after 2 cycles of Cap was documented in 6 patients (15\%). Median time to progression was 2.3 months (range 0.5-45.1) and median overall survival (since start of Cap treatment) was 7.6 months (range 0.745.1). Predominant grade 2 and 3 toxicities (per patient analysis) were hand-foot syndrome $28 \%$ (13\% grade 3); anemia $23 \%$; leg edema $15 \%$; diarrhea $13 \%$; nausea/vomiting $10 \%$, and leukocytopenia 10\%. Conclusion: Single-agent Cap is a safe treatment option for Gem-pretreated patients with advanced PC. Further evaluation of Cap in controlled clinical trials of Gem-pretreated patients with advanced PC is recommended.

Copyright $\odot 2008$ S. Karger AG, Basel

\section{Introduction}

Advanced pancreatic cancer (PC) remains a disease with a very unfavorable prognosis of survival. In 2006, an estimated 33,730 new cases were diagnosed in the United States, with a nearly identical rate of 32,300 deaths from PC [1]. Median survival time for patients with advanced $\mathrm{PC}$ without treatment ranges from 3 to 4 months, where-

Dr. Stefan Boeck, Department of Internal Medicine III, Klinikum Grosshadern Ludwig Maximilians University of Munich

Marchioninistrasse 15, DE-81377 Munich (Germany)

Tel. +49897095 2208, Fax +498970955256

E-Mailstefan.boeck@med.uni-muenchen.de 
as in patients receiving chemotherapy with single-agent gemcitabine (Gem), median survival times between 4.9 and 7.2 months have been reported [2-4]. Single-agent Gem is still regarded as one of the standards of care in the first-line treatment of patients with locally advanced or metastatic PC [2]. To date, only 2 randomized controlled trials have demonstrated a significant prolongation of survival with the use of Gem-based combination therapy with either erlotinib or capecitabine (Cap) $[5,6]$.

The role of second-line treatment after failure of firstline chemotherapy remains controversial in this disease. Preliminary results of a small randomized study comparing best supportive care alone versus chemotherapy with 5-fluorouracil, folinic acid and oxaliplatin plus best supportive care after Gem failure showed a prolongation of median survival by about 2.6 months with chemotherapy (2.3 vs. 4.9 months) [7]. These data correlate well with a recently published study that reported a median survival time of about 1.9 months after failure of first-line Gem in 74 patients with PC (of whom $97 \%$ received no second-line treatment) [8]. Several small phase II trials have also been performed in Gem-pretreated patients with advanced PC: these studies indicate a median survival time of 4.2-6.8 months in patients who received therapy with various anti-cancer agents [9-16]. The largest study, investigating the role of salvage chemotherapy in PC patients after Gem failure, was a randomized trial of over 400 patients. Treatment consisted of either rubitecan (an oral topoisomerase I inhibitor) or the physicians' best choice of treatment or care [17]. In this phase III trial, more than $90 \%$ of the patients were resistant to prior chemotherapy and $70 \%$ were resistant to 2 or more prior regimens. There was no significant difference in median survival between the 2 treatment arms (rubitecan vs. best choice: 3.6 vs. 3.1 months).

Cap (Xeloda ${ }^{\circledR}$; F. Hoffmann-La Roche Ltd., Basel, Switzerland) has shown single-agent activity in the frontline treatment of patients with advanced PC and also a promising efficacy and safety profile in combination with Gem in the phase II setting [18-20]. Two randomized phase III studies have subsequently compared the combination of Gem plus Cap with single-agent Gem: one trial conducted by Herrmann and coworkers [21] used a 3-week regimen of Gem plus Cap and failed to show a significant survival benefit for the combination regimen (7.2 vs. 8.4 months, $\mathrm{p}=0.234$ ). In contrast, preliminary results reported by Cunningham and colleagues [6] showed a significant prolongation of median survival for the Gem plus Cap combination when given at a higher dose intensity in a 4 -week treatment schedule (6.0 vs. 7.4 months; $\mathrm{p}=0.026$ ).
This current study evaluated the efficacy and safety of the oral fluoropyrimidine Cap as a single agent in Gempretreated patients with locally advanced or metastatic PC. For this evaluation, data from Gem-pretreated patients receiving salvage chemotherapy with Cap at our department were collected prospectively. All patients included had progressed after at least 1 full-dose Gem-containing chemotherapy or chemoradiotherapy (CRT) regimen.

\section{Patients and Methods}

\section{Study Design}

Between August 2003 and February 2007, 39 patients with advanced, pretreated PC received Cap chemotherapy at the Department of Internal Medicine III, University of Munich, Germany. All consecutive patients that received at least 1 dose of Cap during this time period were included in the analysis. Data from these patients were collected prospectively and patients gave informed consent to treatment and data collection for statistical analysis and publication.

\section{Patient Population}

All patients eligible for Cap treatment had received at least 1 previous treatment regimen containing full-dose Gem (either as single-agent or combination chemotherapy, or sequentially within a CRT protocol) for the treatment of histologically confirmed advanced PC (stages III and IV). Patients who previously received low-dose Gem as a radiosensitizer only were not defined as 'Gem pretreated' and thus not eligible for this analysis. All patients had progressive disease on imaging after prior Gem-based therapy; each patient that received primary CRT for nonresectable, locally advanced PC progressed by developing distant metastases. Most of the patients undergoing primary CRT received Gem as a radiosensitizer followed by sequential full-dose Gem or Gem-containing chemotherapy. There was no predefined limit on the number of previous therapies. Cap was offered only to patients with a Karnofsky performance status (KPS) $\geq 70 \%$, a life expectancy $\geq 12$ weeks and with adequate hematological, hepatic and renal function. Women who were breastfeeding or pregnant were ineligible for treatment.

Chemotherapy and Supportive Treatment

Chemotherapy with Cap was administered in an outpatient setting. Cap was given orally twice daily at a dose of $1,250 \mathrm{mg} / \mathrm{m}^{2}$ (for patients aged $\geq 65$ years: $1,000 \mathrm{mg} / \mathrm{m}^{2}$ ) for 14 consecutive days followed by 7 days of rest. Treatment cycles were repeated every 21 days. If necessary, dose reductions of Cap were performed according to clinical and laboratory parameters and according to the decision of the treating physician. Chemotherapy was continued until disease progression or unacceptable toxicity. No prophylactic antiemetic treatment was administered. For supportive treatment of the known thrombophilic diathesis that occurs in patients with metastatic PC undergoing chemotherapy [22], patients without a history of thrombosis or pulmonary embolism received - if no contraindications were obvious - a daily prophylactic dose of subcutaneous low-molecular weight heparin. 
Evaluation Criteria for Efficacy and Toxicity

Physical examination (including body weight), KPS, vital signs and blood analysis (hematology and serum chemistry) were performed on day 1 of each cycle. In patients with measurable disease, response to chemotherapy was evaluated with ultrasound, CT/MRI scans or a positron emission tomography-CT scan before treatment and after 2 or 3 cycles of chemotherapy (6 or 9 weeks of treatment, respectively) according to the RECIST criteria [23]. Additionally, serum tumor marker levels of carbohydrate antigen 19-9 (CA19-9) were analyzed on day 1 of every cycle. Reductions in CA19-9 levels of $>20$ and $>50 \%$ in relation to baseline were used for the determination of a biochemical treatment response [24].

Time to progression (TTP) was defined as the time from start of Cap treatment to the date when progressive disease was first confirmed by imaging criteria. In patients where no confirmation of progressive disease by imaging was possible (e.g., clinical disease progression occurred before first staging scan), the date when clinical disease progression was observed and/or when Cap treatment was discontinued was used as time point for the calculation of TTP. Two definitions for analyzing survival time were applied: first, the overall survival (OS) from initial diagnosis of PC to death, and second, OS from the start of Cap treatment to death.

Safety was monitored in all patients who received at least 1 dose of Cap. Toxicity was assessed at the beginning of each cycle according to the National Cancer Institute Common Terminology Criteria for Adverse Events, version 3.0.

\section{Statistical Analysis}

Study endpoints included objective tumor response by imaging, CA19-9 tumor marker response, TTP, OS and toxicity. Baseline patient characteristics and laboratory values were expressed as median values with ranges, and TTP and OS times were determined by the Kaplan-Meier method.

\section{Results}

\section{Patient Characteristics}

In total, 39 patients with histologically confirmed advanced PC were included in this study. Baseline patient characteristics are summarized in table 1 . At treatment initiation with Cap, nearly all patients had metastatic disease (97\%), mainly involving the liver and lung. Pretreatment CA19-9 serum tumor marker levels were elevated (defined as levels $>37 \mathrm{U} / \mathrm{ml}$ ) in 31 patients (80\%); the median CA19-9 baseline level in these patients was 2,438 $\mathrm{U} / \mathrm{ml}$ (range 43-98,833). Regarding previous therapy, 12 patients had initially undergone curative-intent surgery for resectable PC, 9 patients had received previous primary CRT for locally advanced PC and all patients had received prior chemotherapy with full-dose Gem. Seventeen patients (44\%) had been treated with 2 or more previous regimens before Cap was started.
Table 1. Baseline patient characteristics at study entry

\begin{tabular}{|c|c|c|}
\hline Characteristic & Patients $(\mathrm{n}=39)$ & $\%$ \\
\hline Median age, years & $63(42-75)$ & \\
\hline \multicolumn{3}{|l|}{ Gender } \\
\hline Male & 21 & 54 \\
\hline Female & 18 & 46 \\
\hline Median time from diagnosis, months & $9.0(2.5-48.0)$ & \\
\hline \multicolumn{3}{|l|}{ Stage of disease } \\
\hline Locally advanced & 1 & 3 \\
\hline Metastatic & 38 & 97 \\
\hline \multicolumn{3}{|l|}{ Histology } \\
\hline Adenocarcinoma & 37 & 95 \\
\hline Acinar cell carcinoma & 2 & 5 \\
\hline \multicolumn{3}{|l|}{ Tumor grading } \\
\hline G1 & 2 & 5 \\
\hline G2 & 19 & 49 \\
\hline G3 & 18 & 46 \\
\hline \multicolumn{3}{|l|}{ Site of metastasis } \\
\hline Liver & 31 & 79 \\
\hline Lung & 9 & 23 \\
\hline Peritoneum & 2 & 5 \\
\hline Bone & 2 & 5 \\
\hline \multicolumn{3}{|l|}{ KPS } \\
\hline $100 \%$ & 3 & 8 \\
\hline $90 \%$ & 17 & 44 \\
\hline $80 \%$ & 17 & 44 \\
\hline $70 \%$ & 2 & 5 \\
\hline Median pretreatment CA 19-9, U/ml & $2,438(43-98,833)$ & \\
\hline \multicolumn{3}{|l|}{ Previous treatment } \\
\hline Whipple operation & 12 & 31 \\
\hline Prior CRT & 15 & 39 \\
\hline Adjuvant intent & 6 & \\
\hline Palliative intent & 9 & \\
\hline Prior systemic CT & 32 & 82 \\
\hline Prior full-dose Gem & 39 & 100 \\
\hline \multicolumn{3}{|c|}{ Previous treatment regimens (CRT, CT), n } \\
\hline 1 & 22 & 56 \\
\hline 2 & 13 & 33 \\
\hline$\geq 3$ & 4 & 10 \\
\hline
\end{tabular}

Figures in parentheses are ranges.

\section{Treatment and Toxicity}

After a median follow-up of 6.6 months (range 0.745.1), 8 patients are still alive, including 2 patients still on Cap. Chemotherapy with Cap was discontinued mainly due to disease progression (35 patients); 1 patient refused further chemotherapy, and in 1 patient, Cap was discontinued due to toxicity (grade 3 nausea/vomiting). A total of 207 treatment cycles of Cap were administrated during the study, with a median 3 cycles per patient (range 1-36).

Oncology 2007;73:221-227 
Table 2. Hematological and nonhematological toxicity according to NCI-CTCAE (version 3.0)

\begin{tabular}{lclll}
\hline \multirow{2}{*}{ Toxicity } & \multicolumn{2}{l}{ Patients $(\mathrm{n}=39)$} & \\
\cline { 2 - 5 } & grade 1 & grade 2 & grade 3 & grade 4 \\
\hline Leukocytopenia & $9(23)$ & $4(10)$ & 0 & 0 \\
Neutropenia & $1(3)$ & 0 & $1(3)$ & 0 \\
Thrombocytopenia & $11(28)$ & 0 & 0 & 0 \\
Anemia & $13(33)$ & $8(21)$ & $1(3)$ & 0 \\
Hand-foot syndrome & $9(23)$ & $6(15)$ & $5(13)$ & 0 \\
Diarrhea & $7(18)$ & $5(13)$ & 0 & 0 \\
Nausea/vomiting & $9(23)$ & $2(5)$ & $2(5)$ & 0 \\
Mucositis & $8(21)$ & $1(3)$ & 0 & 0 \\
Infection & 0 & $2(5)$ & $1(3)$ & 0 \\
Leg edema & 0 & $6(15)$ & 0 & 0 \\
Skin rash & 0 & $3(8)$ & 0 & 0 \\
Alopecia & $1(3)$ & 0 & 0 & 0 \\
Cerebral ischemia & 0 & 0 & 0 & $1(3)$ \\
\hline
\end{tabular}

Figures in parentheses are percentages. NCI-CTCAE $=\mathrm{Na}-$ tional Cancer Institute Common Terminology Criteria for Adverse Events.

Dose reductions and/or temporary treatment interruptions due to toxicity (mainly hand-foot syndrome and diarrhea) were performed in 11 patients (28\%). Twenty patients $(51 \%)$ received concomitant medication with daily doses of a low-dose low-molecular weight heparin (either therapeutic or prophylactic dosing). Of the 37 patients that discontinued systemic treatment with Cap, 21 patients (57\%) subsequently received further chemotherapy.

All 39 patients were evaluable for toxicity. Hematological and nonhematological toxicity data are summarized in table 2. Cap was generally well tolerated in our patient population. Hematological toxicity was generally mild (grade 1 or 2 severity); no grade 4 hematological toxicity was observed. Regarding the nonhematological toxicities, hand-foot syndrome (51\% of patients), nausea/ vomiting (33\%) and diarrhea (31\%) occurred most frequently. The main grade 3 toxicity observed in this study was hand-foot syndrome in 5 patients (13\%), no patient experienced grade 4 hand-foot syndrome. Only 1 grade 4 toxicity occurred in a patient who suffered from a cerebral ischemia during treatment with Cap. No treatmentrelated deaths were observed.

\section{Response and Survival}

Twenty-seven patients (69\%) were evaluable for response by imaging criteria (table 3 ). One patient received
Table 3. Response to treatment with Cap (RECIST criteria)

\begin{tabular}{lc}
\hline Response by imaging & Patients $(\mathrm{n}=39)$ \\
\hline Evaluable patients & $27(69)$ \\
Partial response & $0(0)$ \\
Stable disease & $15(39)$ \\
Progressive disease & $12(31)$ \\
Disease control rate & $15(39)$ \\
\hline
\end{tabular}

Figures in parentheses are percentages.

only 1 cycle of Cap before changing treatment because of grade 3 nausea; no assessment of response was therefore possible. Two patients did not have measurable disease and a further 9 patients (23\%) showed clinically rapid disease progression and did not undergo radiological evaluation and confirmation of progressive disease. No complete or partial remission was observed. Disease stabilization was achieved in 15 of the 39 patients analyzed, resulting in a disease control rate of $39 \%$. One patient with a poorly differentiated (G3) acinar cell carcinoma of the pancreas corpus with multiple liver metastases experienced long-term (>45.1 months) disease stabilization by RECIST criteria. Additionally, a positron emission tomography-CT scan showed a nearly complete metabolic response of the liver lesions to treatment with Cap in this patient.

Twenty-eight of 31 patients with elevated baseline CA19-9 levels were evaluated for a CA19-9 tumor marker response. A CA19-9 decline of $>20 \%$ after 2 cycles of chemotherapy (6 weeks) was observed in 6 patients $(15 \%)$, and a decline in CA19-9 values by $>50 \%$ was observed in 3 patients (8\%). All patients with a CA19-9 decline $>20 \%$ showed stable disease on imaging.

The estimated median TTP was 2.3 months (range 0.5-45.1); the Kaplan-Meier plot for TTP is shown in figure 1. At the time of final analysis in May 2007, 31 patients had died. Median OS since the start of Cap treatment was estimated to be 7.6 months (range 0.7-45.1); the KaplanMeier plot for OS is shown in figure 2. The median survival time calculated from initial diagnosis of $\mathrm{PC}$ to death was 19.2 months (range 4.2-60.4).

\section{Discussion}

There is increasing evidence that selected patients with advanced PC may benefit from second-line chemotherapy after failure of first-line Gem or Gem-based 


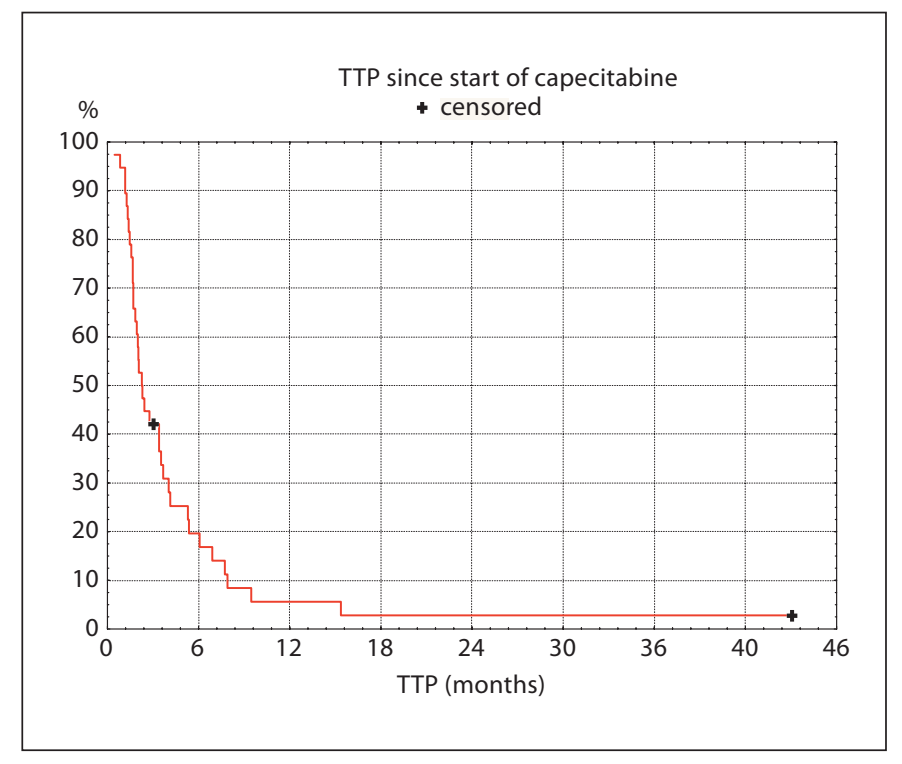

Fig. 1. Estimated TTP (median 2.3 months, range 0.5-45.1).

treatment regimens. Based on currently available data, a median survival time of about 1.9 to 2.3 months after failure of Gem can be expected without further chemotherapy $[7,8]$. Several phase II trials using different treatment regimens suggest an improved median survival time (from the start of second-line chemotherapy) in the range of 4.2 to 6.8 months [16]. Nevertheless, these results must still be regarded carefully, and it should be kept in mind that nonrandomized phase II trials often include a possible selection bias. The latest version of the National Comprehensive Cancer Network guidelines for pancreatic adenocarcinoma recommends a second-line chemotherapeutic treatment after failure of first-line Gem in selected patients using, for example, single-agent Cap or a FOLFOX (5-fluorouracil/leucovorin and oxaliplatin)like regimen [25]. However, to date, no data from clinical trials using Cap monotherapy as salvage treatment after Gem failure are available. Only 2 groups reported results from second-line phase II trials using a combination regimen of Cap plus erlotinib and Cap plus oxaliplatin, respectively $[13,26]$.

The prospective study data reported here demonstrate that single-agent Cap is a safe treatment option for this patient population. The toxicity data in Gem-pretreated patients did not show any marked differences compared with the first-line study published by Cartwright and colleagues [18] that used the same Cap dose schedule as our study. However, the overall incidence of hand-foot syn-

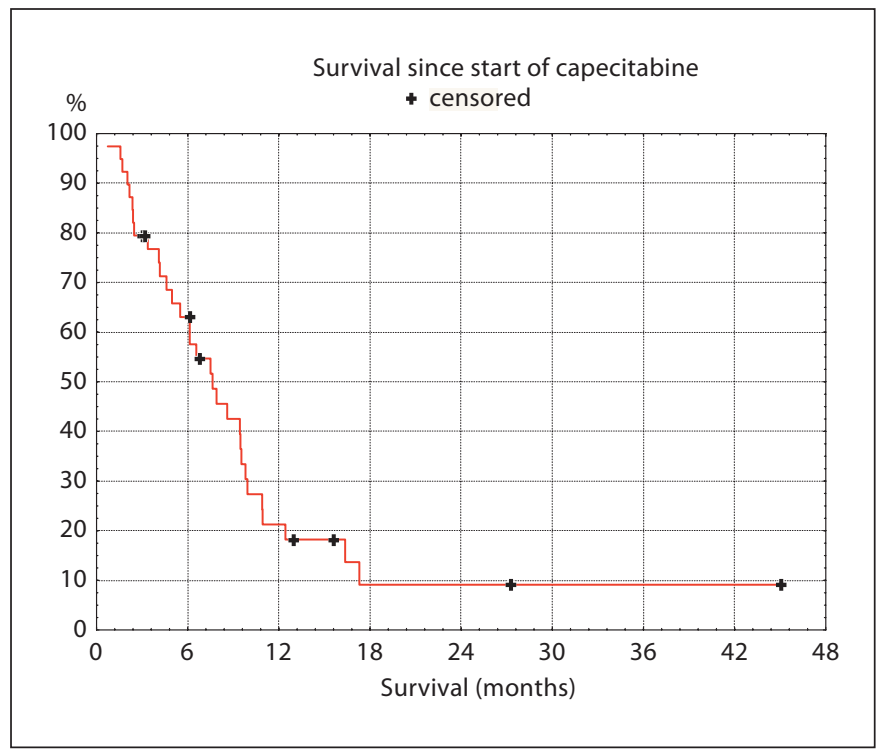

Fig. 2. Estimated OS (median 7.6 months, range 0.7-45.1).

drome in our patient population was $51 \%$, with 5 patients (13\%) experiencing grad 3 toxicity. Cartwright et al. [18] reported an overall incidence of $52 \%$ for hand-foot syndrome in their first-line phase II study, with a grade 3 rate of $17 \%$. As the patients' quality of life may be significantly affected by this clinically relevant toxicity, one might also consider a reduced dose of Cap (e.g., $1,000 \mathrm{mg} / \mathrm{m}^{2}$ on days 1-14 every 3 weeks for all patients independent of their age) when used as a salvage monotherapy after Gem failure. If Cap is combined with a second drug, e.g., erlotinib [13] or oxaliplatin [26], in this setting, toxicity should also be regarded carefully; especially if the 2-drug regimen may result in an overlapping toxicity profile (like skin toxicity or diarrhea, respectively).

Regarding efficacy, single-agent Cap produced a disease control rate of $39 \%$ with no objective responses. These data appear inferior compared with other phase II trials, mainly using oxaliplatin-based combination chemotherapy, in the second-line setting $[12,14,15]$. However, our study population might represent a group of patients with poor prognostic features $(100 \% \mathrm{Gem}$-pretreated patients, $97 \%$ with metastatic disease, $49 \%$ with a KPS $<90 \%$ and $44 \%$ who had received 2 or more treatment regimens before study entry). Based on these baseline patient characteristics, a median TTP of 2.3 months (about 10 weeks) and an OS of 7.6 months (about 32 weeks) from the start of Cap treatment appear similar to the results of phase II studies investigating combination chemothera- 
py in this patient population [16]. Furthermore, preliminary results of a randomized second-line trial after Gem failure (comparing 5-fluorouracil/folinic acid vs. 5-fluorouracil/folinic acid plus oxaliplatin) reported a median TTP of about 1.9 months ( 8 weeks) for the reference arm with 5-fluorouracil/folinic acid [27]. However, interstudy comparisons of survival results can be difficult to perform correctly: patients may receive further treatment after failure of the study therapy (57\% of patients received further chemotherapy after Cap failure in this study) and also because of differences in important patient prognostic factors. The current study data are derived from a single, high-volume German center. Therefore, it is likely that the survival results represent a 'positive' selection bias (also with regard to the observed median OS of 19.2 months from first diagnosis of PC).

This study also included 2 patients with the rare histology of a pancreatic acinar cell carcinoma. These patients do not only have different histological features compared with patients with (ductal) adenocarcinoma, they also seem to have a different prognosis $[28,29]$. Both patients with metastatic acinar cell carcinoma in this study experienced prolonged disease control during treatment with Cap (TTP of 9.5 and $>45.1$ months, respectively), and they showed a favorable survival prognosis with an OS (from initial diagnosis) of 60.4 and $>48.1$ months, respectively.

To date, it remains an unsolved and important clinical question which treatment should be offered to a patient with advanced PC who requests further chemotherapy after Gem failure. In our opinion, patients for second-line therapy should be selected carefully based on known prognostic factors for this disease [8, 30]. For example, KPS may serve as a useful and convenient clinical tool. There is increasing evidence that pretreatment KPS is a useful prognostic factor in the first-line setting, and that it possibly also has the potential to select patients for different treatment strategies (e.g., single-agent vs. combination chemotherapy) [21,30]. More recently, KPS was identified as an independent prognostic factor for OS and progression-free survival in PC patients that failed prior Gem treatment $[8,31]$. Thus, a clinical investigation of these important issues regarding patient selection and treatment individualization is strongly recommended. Only well-designed, randomized, controlled clinical trials will help to answer the current - and clinically extremely relevant - questions concerning the benefit of second-line chemotherapy. Based on the data reported here, and also with regard to the encouraging results from randomized trials in the first-line setting, Cap should be included in these investigations either as a single agent or as part of a combination regimen $[13,26$, 32].

\section{Acknowledgements}

The authors would like to thank Matthias Wolff for his statistical support, as well as Silke Lorenz and Helga Kaiser for their administrative assistance.

\section{References}

1 Ozols RF, Herbst RS, Colson YL, et al: Clinical cancer advances 2006: major research advanced in cancer treatment, prevention, and screening - a report from the American Society of Clinical Oncology. J Clin Oncol 2007;25:146-162.

2 Burris HA, Moore MJ, Andersen J, et al: Improvements in survival and clinical benefit with gemcitabine as first-line therapy for patients with advanced pancreas cancer: a randomized trial. J Clin Oncol 1997;15:24032413.

3 Heinemann V: Gemcitabine in the treatment of advanced pancreatic cancer: a comparative analysis of randomized trials. Semin Oncol 2002;29(suppl 20):9-16.

4 Hochster HS, Haller DG, de Gramont A, et al: Consensus report of the International Society of Gastrointestinal Oncology on therapeutic progress in advanced pancreatic cancer. Cancer 2006;107:676-685.
5 Moore MJ, Goldstein D, Hamm J, et al: Erlotinib plus gemcitabine compared with gemcitabine alone in patients with advanced pancreatic cancer: a phase III trial of the $\mathrm{Na}$ tional Cancer Institute of Canada Clinical Trials Group. J Clin Oncol 2007;25:19601966.

6 Cunningham D, Chau I, Stocken D, et al: Phase III randomised comparison of gemcitabine (GEM) versus gemcitabine plus capecitabine (GEM-CAP) in patients with advanced pancreatic cancer (abstract PS11). Eur J Cancer 2005;3(suppl):4.

7 Oettle H, Pelzer U, Stieler J, et al: Oxaliplatin/folinic acid/5-fluorouracil (24 h) (OFF) plus best supportive care versus best supportive care alone (BSC) in second-line therapy of gemcitabine-refractory advanced pancreatic cancer (CONKO 003) (abstract 4031). Proc Am Soc Clin Oncol 2005;23: $315 \mathrm{~s}$
8 Nakachi K, Furuse J, Ishii H, Suzuki E, Yoshino M: Prognostic factors in patients with gemcitabine-refractory pancreatic cancer. Jap J Clin Oncol 2007;37:114-120.

9 Oettle H, Arnold D, Esser M, Huhn D, Riess $\mathrm{H}$ : Paclitaxel as weekly second-line therapy in patients with advanced pancreatic carcinoma. Anticancer Drugs 2000;11:635-638.

10 Milella M, Gelibter A, Di Cosimo S, et al: Pilot study of celecoxib and infusional 5-fluorouracil as second-line treatment for advanced pancreatic carcinoma. Cancer 2004; 101:133-138.

11 Cantore M, Rabbi C, Fiorentini G, et al: Combined irinotecan and oxaliplatin in patients with advanced pre-treated pancreatic cancer. Oncology 2004;67:93-97.

12 Tsavaris N, Kosmas C, Skopelitis H, et al: Second-line treatment with oxaliplatin, leucovorin and 5-fluorouracil in gemcitabine-pretreated advanced pancreatic cancer: a phase II study. Invest New Drugs 2005;23:369-375. 
13 Kulke MH, Blaszkowsky LS, Ryan DP, et al: Capecitabine plus erlotinib in gemcitabinerefractory advanced pancreatic cancer. J Clin Oncol 2007;25:4787-4792.

14 Demols A, Peeters M, Polus M, et al: Gemcitabine and oxaliplatin (GEMOX) in gemcitabine refractory advanced pancreatic adenocarcinoma: a phase II study. Br J Cancer 2006;94:481-485.

15 Reni M, Pasetto L, Aprile G, et al: Raltitrexed-eloxantin salvage chemotherapy in gemcitabine-resistant metastatic pancreatic cancer. Br J Cancer 2006;94:785-791.

16 Boeck S, Weigang-Köhler K, Fuchs M, et al: Second-line chemotherapy with pemetrexed after gemcitabine failure in patients with advanced pancreatic cancer: a multicenter phase II trial. Ann Oncol 2007;18:745-751.

17 Jacobs AD, Burris HA, Rivkin S, et al: A randomized phase III study of rubitecan (ORA) vs best choice (BC) in 409 patients with refractory pancreatic cancer. Report from a North-American multi-center study (abstract 4013). Proc Am Soc Clin Oncol 2004; 22:316s.

18 Cartwright TH, Cohn A, Varkey JA, et al: Phase II study of oral capecitabine in patients with advanced or metastatic pancreatic cancer. J Clin Oncol 2001;20:160-164.

19 Scheithauer W, Schüll B, Ulrich-Pur H, et al: Biweekly high-dose gemcitabine alone or in combination with capecitabine in patients with metastatic pancreatic adenocarcinoma: a randomized phase II trial. Ann Oncol 2004;14:97-104.
20 Stathopoulos GP, Syrigos K, Polyzos A, et al: Front-line treatment of inoperable or metastatic pancreatic cancer with gemcitabine and capecitabine: an intergroup, multicenter, phase II study. Ann Oncol 2004;15:224229.

21 Herrmann R, Bodoky G, Ruhstaller T, et al: Gemcitabine plus capecitabine compared with gemcitabine alone in advanced pancreatic cancer. A randomized, multicenter, phase III trial of the Swiss Group for Clinical Cancer Research and the Central European Cooperative Oncology Group. J Clin Oncol 2007;25:2212-2217.

22 Blom JW, Osanto S, Rosendaal FR: High risk of venous thrombosis in patients with pancreatic cancer: a cohort study of 220 patients. Eur J Cancer 2006;42:410-414.

23 Therasse P, Arbuck SG, Eisenhauer EA, et al New guidelines to evaluate the response to treatment in solid tumors. European Organization for Research and Treatment of Cancer, National Cancer Institute of the United States, National Cancer Institute of Canada. J Nat Can Inst 2000;92:205-216.

24 Boeck S, Stieber P, Holdenrieder S, Wilkowski R, Heinemann V: Prognostic and therapeutic significance of carbohydrate antigen $19-9$ as tumor marker in patients with pancreatic cancer. Oncology 2006;70:255-264.

25 Tempero MA, Behrman S, Ben-Josef E, et al: Pancreatic adenocarcinoma. Clinical practice guidelines in oncology. J Natl Compr Canc Netw 2005;3:598-626 (for update, version V.I. 2008, see www.nccn.org).
26 Xiong HQ, Wolff RA, Hess KR, et al: A phase II trial of oxaliplatin plus capecitabine (xelox) as second line therapy for patients with advanced pancreatic cancer (abstract 4119). Proc Am Soc Clin Oncol 2006;24:207s.

27 Riess H, Pelzer U, Stieler J, et al: A randomized second line trial in patients with gemcitabine refractory advanced pancreatic cancer - CONKO 003 (abstract 4517). Proc Am Soc Clin Oncol 2007;25:201s.

28 Holen KD, Klimstra DS, Hummer A, et al: Clinical characteristics and outcome from an institutional series of acinar cell carcinoma of the pancreas and related tumors. J Clin Oncol 2002;20:4673-4678.

29 Kitagami H, Kondo S, Hirano S, et al: Acinar cell carcinoma of the pancreas: clinical analysis of 115 patients from pancreatic cancer registry of Japan pancreas society. Pancreas 2007;35:42-46.

30 Boeck S, Hinke A, Wilkowski R, Heinemann V: Importance of performance status for treatment outcome in advanced pancreatic cancer. World J Gastroenterol 2007;13:224227.

31 Mancuso-Petricca A, Saletti P, Tronconi $\mathrm{MC}$, et al: The efficacy of fluoropyrimidinebased second line chemotherapy in advanced and metastatic pancreatic cancer: results of an ongoing Italian/Swiss multicenter survey (abstract 1131P). Ann Oncol 2006;17(suppl 9):ix323.

32 Fine RL, Fogelman DR, Schreibman SM, et al: The gemcitabine, docetaxel, and capecitabine (GTX) regimen for metastatic pancreatic cancer: a retrospective analysis. Cancer Chemother Pharmacol 2008;61:167-175. 